\title{
Numerical Modeling of the Exposure on Radiofrequency Radiation of Marine Mammal Observers During Their Shift: A Case Study
}

\author{
Christos C. Spandonidis ${ }^{1 *}$, Kyriakoula Arvaniti ${ }^{2}$ \\ ${ }^{1}$ Prisma Electronics SA, Leof. Poseidonos 42, Kallithea 17675, Greece \\ ${ }^{2}$ EOS Energy Solutions and Support, Kemp House 160 City Road, London EC1V 2NX, UK
}

Corresponding Author Email: c.spandonidis@prismael.com

https://doi.org/10.18280/ijsse.100605

Received: 19 October 2020

Accepted: 2 December 2020

\section{Keywords:}

numerical modeling, risk assessment, decision support, occupational health, marine mammal observers

\begin{abstract}
Following the standard numerical modeling approach for Electromagnetic Field (EMF) radiation exposure prediction, we intend to provide an analytical framework to Marine Mammal Observers (MMOs) for dynamic risk assessment; enhancing thus occupational health and safety awareness. The analysis is based on power levels and antenna characteristics reported by MMOs for two systems (VHF and UHF) located close to the working environment. Whilst occupational exposure limits apply for MMOs, as for the rest crew (seismic and maritime), evaluation of exposure levels against general public limits is presented as well. At present we have restricted our study to single-source radiation, as well as we did not consider any irregularities due to system malfunction. The worst-case scenario of continuous RF transmission was considered. Risk assessment indicated regions where radiation exposure is higher than the permissible limits. Uncertainty due to the operational environment is inserted in methodology using an uncertainty coefficient. A list of control measures is proposed, to support both MMO's and Operators' decision making.
\end{abstract}

\section{INTRODUCTION}

Marine mammals use sound to communicate, locate prey, avoid predators, or sense their environment [1]. Seismic operations constitute a significant possible threat for marine mammals, since operations usually run for long periods, emitting intense sound mainly of low frequencies. Effects can impact the animals' biology, physiology, and mask biologically significant sounds [2]. During seismic operations - as part of the mitigation effort- sightings, behavioral, and species identification data are usually collected from qualified Marine Mammal Observers (MMO). According to Joint Nature Conservation Committee [3], an MMO is the crew member responsible for monitoring, through visual watches, the appearance of the marine mammals within the area of interest, during seismic surveys, and for provision of the appropriate mitigation measures upon their detection. It is a common experience for MMOs to be located during their shift on suitable spots, on-board the source vessel, enabling the best view of the mitigation zone [3]. Even though beneficial for visibility, these spots are frequently located next to antennas used for radiofrequency (RF) communications. While specific plans by operators and restrictions by class societies are applied to almost every seismic vessel [4], it is not rare for an MMO to perform his duties in the close vicinity of an RF antenna. A coherent framework for the study of the exposure on radiofrequency radiation of marine mammal observers during their shift has not been set up yet. Most studies (e.g. [5]) are related to different environments, not strictly applicable to the maritime field. As a result, up to now in the seismic industry field, the application of numerical modeling approaches for this important topic is non - existent.
In the current work, we present a numerical modeling method for the identification of electromagnetic radiation exposure, based on information taken from the vessel. The motivation behind the current effort comes from a desire to understand better involved risks and to provide a framework for enhancing occupational health and safety awareness for this category of the seismic crew. The focus has been given on the inclusion of uncertainty introduced by the operational environment, using an uncertainty coefficient. Our main goal is to provide an analytical framework [6] for performing risk assessment by a) identification of the hazards, b) calculation of the critical distances from the antenna, after which the exposure extends the permissible limits decide about the impact on health, and c) decision of the proper control measures (Figure 1).

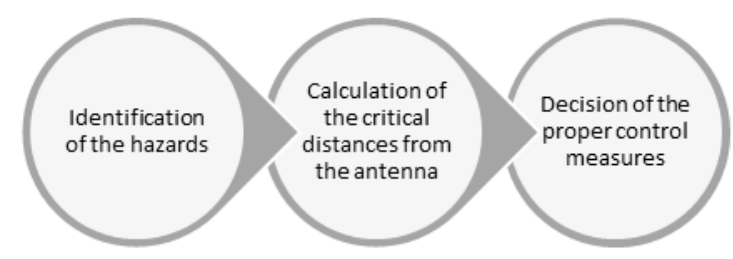

Figure 1. The proposed framework for performing risk assessment and mitigation measures control

Working towards that direction, in Section 2, background information about physics, impact on health, and active legislation is critically presented. In Section 3, after a short description of antenna radiation regions and graphical 
presentation of the safety zones, the main steps of the methodology, as well as discussion about its limitations, are provided. In Section 4 we present the analysis results for the performance assessment based on two reported cases of a UHF and a VHF voice transmitting systems. Finally, in Section 5, we discuss the key results of the study.

\section{BACKGROUND}

Due to the presence of electric and magnetic fields within an electromagnetic wave, plane wave power density $S$ (expressed in $\mathrm{mW} / \mathrm{cm}^{2}$ or $\mathrm{W} / \mathrm{m}^{2}$ ) is commonly used for the characterization of RF electromagnetic fields [5]. The density in the far-field (typically some $\mathrm{cm}$ far from the antenna) may be calculated by the Electric field strength:

$$
S=\frac{E^{2}}{377}
$$

where, $E$ is expressed in $V / m$. The two major categories of Electromagnetic radiation are ionizing and non-ionizing radiation. The former is the most dangerous when it comes to human health as, due to the high energy, it can create ionized atoms (removing the electrons from their orbit). The Radiofrequency $(3 \mathrm{kHz}-300 \mathrm{GHz})$, is part of the latter category, non-ionizing, that does not have sufficient energy to ionize the atoms. RF is mainly used for communication purposes and is further divided into several subcategories based on signal frequency/wavelength, as shown in Table 1.

Table 1. Radiofrequency spectrum

\begin{tabular}{ccc}
\hline $\begin{array}{c}\text { Frequency } \\
\text { category }\end{array}$ & Frequency range & wavelengths \\
\hline Very Low (VLF) & $3 \mathrm{kHz}-30 \mathrm{kHz}$ & $100 \mathrm{~km}-10 \mathrm{~km}$ \\
Low (LF) & $30 \mathrm{kHz}-300 \mathrm{kHz}$ & $10 \mathrm{~km}-1 \mathrm{~km}$ \\
Medium (MF) & $300 \mathrm{kHz}-3 \mathrm{MHz}$ & $1 \mathrm{~km}-100 \mathrm{~m}$ \\
High (HF) & $3 \mathrm{MHz}-30 \mathrm{MHz}$ & $100 \mathrm{~m}-10 \mathrm{~m}$ \\
Very High (VHF) & $30 \mathrm{MHz}-300 \mathrm{MHz}$ & $10 \mathrm{~m}-1 \mathrm{~m}$ \\
Ultra High (UHF) & $300 \mathrm{MHz}-3 \mathrm{GHz}$ & $1 \mathrm{~m}-100 \mathrm{~mm}$ \\
Super High (SHF) & $3 \mathrm{GHz}-30 \mathrm{GHz}$ & $100 \mathrm{~mm}-10 \mathrm{~mm}$ \\
Extremely High & $30 \mathrm{GHz}-300 \mathrm{GHz}$ & $10 \mathrm{~mm}-1 \mathrm{~mm}$ \\
(EHF) & \\
\multicolumn{3}{c}{ source: IEEE Standard 521 [6]. } \\
\hline
\end{tabular}

Despite, their great importance, RF systems have been linked to a variety of adverse health outcomes including tissue heat, stimulation of peripheral nerves (short-term exposure) [7] as well as adult leukemia, brain tumors, and DNA damage (long-term exposure) [8].

In that framework, Permissible Exposure Levels (PELs), have been developed by various National and International Organizations, (e.g. [5, 7, 9, 10]). Application of appropriate administrative or engineering controls is required for areas where exposure exceeds the corresponding PELs. It is noted that the theoretical approach must always be followed by experimental measurements according to Electronic Communications Committee's (ECC) guidelines [11, 12]. When it comes to the maritime industry, most commercial ship Electromagnetic Compatibility (EMC) regulations of marine classification societies in the US, Canada, and the European Union follow the IEC60945 [13] standard for the evaluation of a product from a safety perspective (e.g. $[14,15])$.

It has to be noted though that pathologies from long-term exposure (e.g. cancer) were not considered to be established, thus not taken into account during guideline development [7]. As such the Specific Absorption Rate (SAR expressed in $W / \mathrm{kg}$ ), a value that corresponds to the relative amount of RF energy absorbed by a certain amount of tissues of the exposed body has been adopted as threshold values for the calculation of the limits.

\section{METHODOLOGY}

In the current work, the modeling approach described in standard EN 62232:2017 [16] was used. The uncertainty factor $u$, related to the operational environment was further introduced to the model, as presented by FCC [5]. The worstcase scenario [12] was adopted; that is the maximum power levels and antenna gains were evaluated as well as continuous (for at least 6 minutes) power output was assumed. The calculated values were compared against the appropriate ICNIRP reference values, which for the UHF system were lower. Initially, calculation of the exposure regions (antenna regions) is performed, based on the specifications of the RF system under evaluation. Based on these calculations the appropriate Electromagnetic Field calculation method is followed, as described in the following paragraphs.

\subsection{Antenna regions}

Following Annex A of EN 62232:2017 [17] the antenna regions are divided into three categories: a) Far-field region, b) radiating near-field region and c) reactive near-field region.

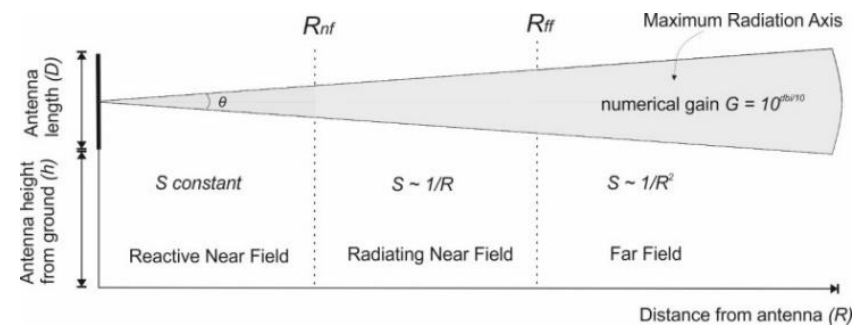

Figure 2. Antenna regions

Inside the reactive near-field region, the electric and magnetic fields, seem to be in-depended of each other. The modeling approach is very challenging. On the other hand, inside radiating near field and the far-field region the power flux density inversely depends on distance and the square of the distance, respectively. Figure 2, graphically presents the determination of the three regions. It is noted that $R(m)$ is the distance from the antenna, $\mathrm{D}(m)$ the length of the antenna. $R_{f f}$, $R_{n f}$ the distances from the antenna that define the far field and reactive near field regions:

$$
R_{f f}=\frac{2 D^{2}}{\lambda} \text { and } R_{n f}=\frac{\lambda}{4}
$$

\subsection{Electromagnetic field calculation method}

EN 62232:2017 [18] describes an evaluation method and possible alternatives for each region. In the current work the following methods were applied:

For the far-field region, the power flux density (S) from the transmitting antenna and is given by: 


$$
S_{f f}=\frac{P_{i n} G}{4 \pi R^{2}} u^{2}
$$

where, $P_{i n}$ is the antenna's input power density (maximum), $G$ is antenna's numerical gain and $u$ is a coefficient that describes the impact of the specific environment into which the system operates. The power density in a point out of the maximum radiation axis is given by:

$$
S=\frac{P_{\text {in }} \cdot G(\theta)}{4 \pi R^{2}} u^{2}
$$

$G(\theta)$ is provided by the antenna manufacturer. Inside the radiating near-field, the cylindrical wave model has been adopted and the power flux density is given by:

$$
\mathrm{S}=\frac{180}{\pi D R} \frac{P_{\text {in }}}{\delta} u^{2}
$$

where, $\delta$ is the angle in degrees defining the $-3 \mathrm{~dB}$ beamwidth of a directional antenna (for an omnidirectional antenna is equal to 360 ).

The far-field and cylindrical wave models give the same power flux density value for distance $R_{c}$ :

$$
R_{c}=\frac{G D}{2} \frac{\delta}{360}
$$

In the region between $R_{f f}$ and $R_{n f}$ the highest calculated compliance perimeter of cylinder/far-field models may be applied.

In all cases, the region inside the reactive near field (described in the following paragraph) was assumed to be a restricted area both for the general public and for the crew.

To validate the model's accuracy, Spandonidis et al. [19] performed a series of on-site measurements. Measured safety zones were evaluated against their numerical calculated counterparts. It was proved that the prediction of the safety zones using this model is in good agreement with the actual situation when uncertainty coefficient $(u)$ close to 1.6 was used. It must be noted that their measurements were conducted inside a working environment similar to that of a vessel.

\subsection{Safety zones calculation method}

Table 2. Permissible Exposure Levels (PEL) for most common directives

\begin{tabular}{cccc}
\hline $\begin{array}{c}\text { Exposure } \\
\text { Category }\end{array}$ & Frequency Range & $\begin{array}{c}\text { FCC } \\
\left(\mathbf{m W} / \mathbf{c m}^{2}\right)\end{array}$ & $\begin{array}{c}\text { ICNIRP } \\
\left(\mathbf{W} / \mathbf{m}^{2}\right)\end{array}$ \\
\hline \multirow{2}{*}{ General } & VHF & 0.2 & 2 \\
Public & UHF up to 400 MHz & $f / 1500$ & 2 \\
& UHF 0.4 - 1.5 GHz & $f / 1500$ & $f / 200$ \\
Occup. & VHF & 1 & 10 \\
& UHF up to 400 MHz & $f / 300$ & 10 \\
& UHF 0.4 - 1.5 GHz & $f / 300$ & $f / 40$ \\
& $* \mathrm{f}=$ frequency in MHz: & \\
\hline
\end{tabular}

Table 2 presents the Permissible Exposure Levels (PEL) recommended by the FCC and ICNIRP, for the cases of VHF and UHF RF systems. As shown both directives divide the Exposure categories into a) Occupational and b) General public exposure. The essence of this categorization is the controllability of the exposure by the former category which most of the time is aware and even responsible for the operation of the RF systems. In contrast, members of the latter category are unaware of their exposure [7]. In what follows, even though MMOs belong to the first category, evaluation of the calculated value against both limits are performed. $R_{\text {red }}$ and $R_{\text {yellow }}$ are the distances from the antenna where the calculated value of power flux density is equal to the professionals and general public limit, respectively. $h_{\text {red }}$ and $h_{\text {yelow }}$ are used to determine appropriate engineering corrective actions.

Inside a red zone, (calculated power density larger than the $1^{\text {st }}$ category's PELS) entrance is not allowed when the RF system is transmitting. Inside the yellow zone (calculated power density larger than $2^{\text {nd }}$ but lower than $1^{\text {s }}$ category's PELs) only technical crew members are allowed, while there are no entrance restrictions inside the green zone where calculated power density is lower than $2^{\text {nd }}$ category's PELs.

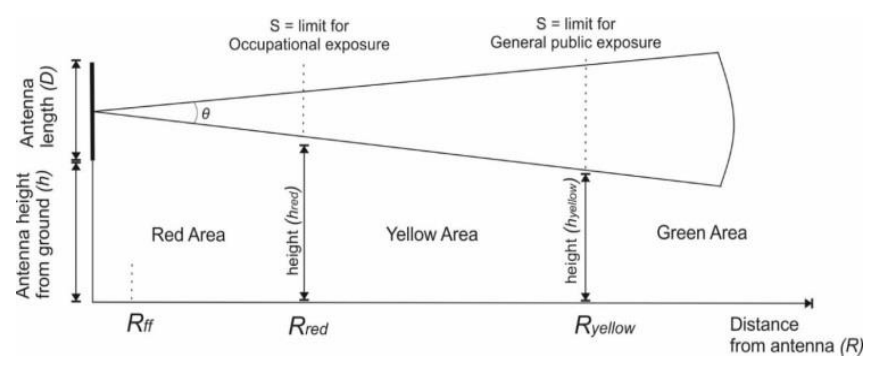

Figure 3. Safety zone categories

Figure 3 graphically presents the safety zone categories. $R_{\text {red }}$ and $R_{\text {yellow }}$ are the distances from the antenna where the calculated value of power flux density is equal to the professionals and general public limit, respectively. $h_{\text {red }}$ and $h_{\text {yelow }}$ are used to determine appropriate engineering corrective actions.

\subsection{Methodology limitations- restrictions}

A general drawback of this (and every other) modeling method is that it does not consider irregularities due to systems malfunction, such as corroded connectors or mismatched loads, etc., which have been proven to be crucial when it comes to health and safety. Also, the transmitting power of the RF systems is assumed to be continuous; thus, power flux density is constant over time.

It is noted that while in the current work demonstration of the methodology through a single RF source is provided, the method can be used when multiple antennas are co-located in the same area (as is the case in a vessel). In that case, the sum of all individual calculated power flux density values is compared with relevant PELs.

\section{NUMERICAL RESULTS}

The aim of this section is the demonstration of the proposed methodology for numerical evaluation of the electromagnetic exposure on MMOs, due to the operation of the vessel's radiotelephony system. Figure 4 depicts the antennas (VHF and UHF), located in the most common spot for visual watches onboard a seismic vessel. Technical specifications of the corresponding RF systems are summarized in Table 3.

Table 4 summarises the estimated PELs for the systems under evaluation. Furthermore, the calculated antenna regions for each case are summarized in Table 5 . 


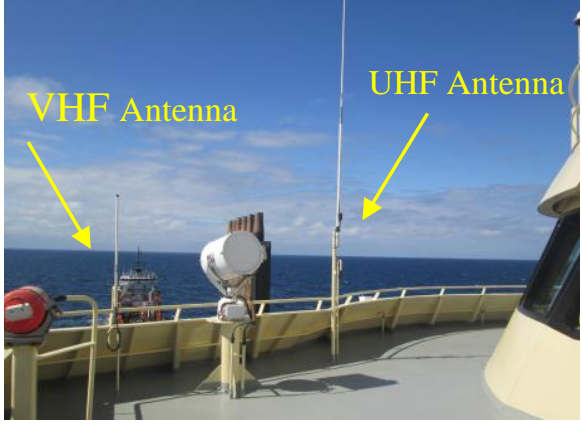

Figure 4. Radiotelephony system's antennas onboard. Marine Mammal Observers

Table 3. Technical specification of UHF/VHF systems

\begin{tabular}{ccc}
\hline System Specifications & UHF & VHF \\
\hline Transmit Power (W) & 25 & 25 \\
Frequency (MHz) & 452 & 245 \\
Antenna Gain (dBi) & 2.15 & 2 \\
E-plane Beam width (degrees) & 120 & 120 \\
H-plane Beam width (degrees) & 360 & 360 \\
Antenna Length (m) & 0.9 & 1,5 \\
Antenna Height (m) & 1.2 & 1 \\
* Attenuation losses through the cable aren't considered \\
\hline
\end{tabular}

Table 4. PELs for UHF RF systems under evaluation

\begin{tabular}{ccc}
\hline $\begin{array}{c}\text { Exposure } \\
\text { Category }\end{array}$ & $\begin{array}{c}\text { Frequency } \\
\text { Range }\end{array}$ & $\begin{array}{c}\text { ICNIRP } \\
\left(\mathbf{m a w} / \mathbf{c m}^{2}\right)\end{array}$ \\
\hline General Public & UHF & 2.26 \\
Occup. & VHF & 2 \\
& UHF & 11.3 \\
$*$ calculated values for power flux density & 10 \\
\hline
\end{tabular}

Table 5. Calculation of safety zone limits

\begin{tabular}{cccc}
\hline Case & $\mathbf{R}_{\mathbf{n f}}$ & $\mathbf{R}_{\mathbf{c}}$ & $\mathbf{R}_{\mathbf{f f}}$ \\
\hline UHF $(\mathrm{u}=1)$ & 0.16 & 0.73 & 2.4 \\
$\operatorname{UHF}(\mathrm{u}=1.6)$ & 0.16 & 0.73 & 2.4 \\
VHF $(\mathrm{u}=1)$ & 0.47 & 1.19 & 2.37 \\
$\operatorname{VHF}(\mathrm{u}=1.6)$ & 0.47 & 1.19 & 2.37 \\
* All values in meters $(m)$ & \\
\hline
\end{tabular}

Following the described methodology, the next step involves the calculation of power flux density at different distances (Eqns. (3) - (6)) from the antenna. Figures 5 and 6 illustrate the correlation between power flux density and distance from the antenna. Initially, a coefficient $u$ value of 1 was used. This value represents an operation in open space and is commonly used in most of the relevant efforts. In a more realistic scenario (onboard environment), the uncertainty coefficient would be higher than 1 . A value of 1.6 has been reported [12] for more crowded environments (e.g. urban, military, vessel).

Based on these values we can estimate the safety zones of each system. Figure 7, graphically presents these results for the UHF system $(u=1)$. As shown the red zone is extended to $0.4 \mathrm{~m}$, while the yellow zone to $1.95 \mathrm{~m}$ from the antenna $\left( \pm 30^{\circ}\right.$ from the main radiation axis in both cases).

For $u=1.6$, the red zone extends to $1 \mathrm{~m}$ and the yellow zone to $2 \mathrm{~m}$ from the antenna. Similarly, Figure 8 presents the calculated zones for the VHF system $(u=1)$. The model estimated that the red zone extends to $0.47 \mathrm{~m}$ while the yellow zone to $1.32 \mathrm{~m}$ from the antenna.

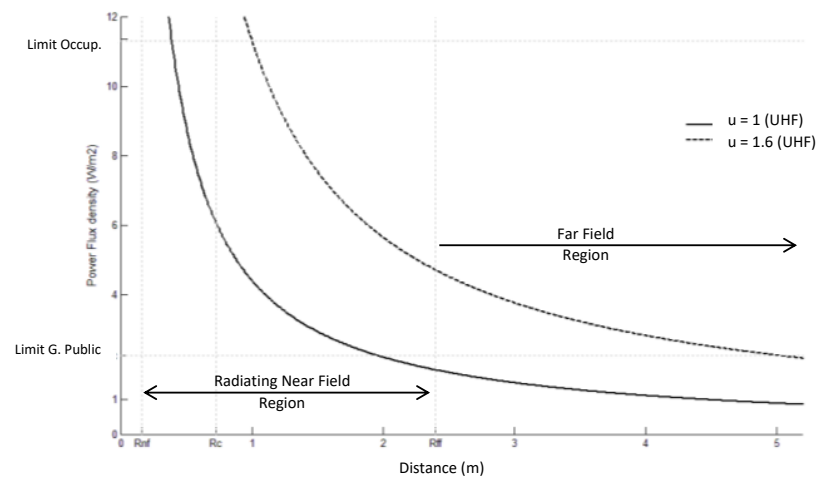

Figure 5. Estimated results for power flux density against distance from the antenna for UHF system

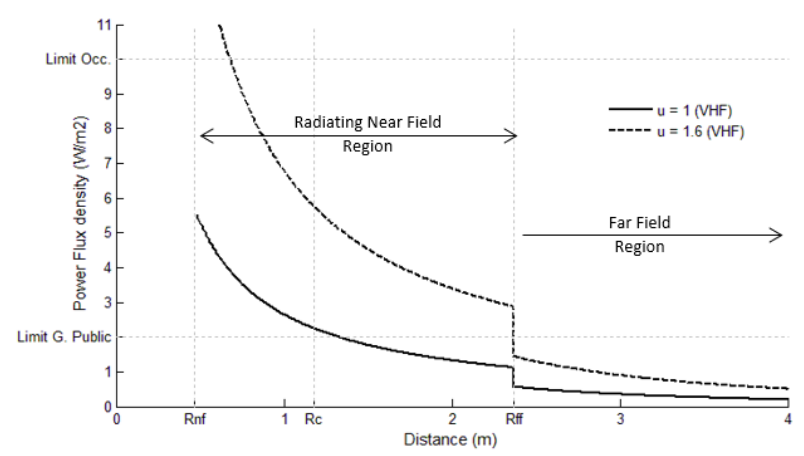

Figure 6. Estimated results for power flux density against distance from the antenna for the VHF system

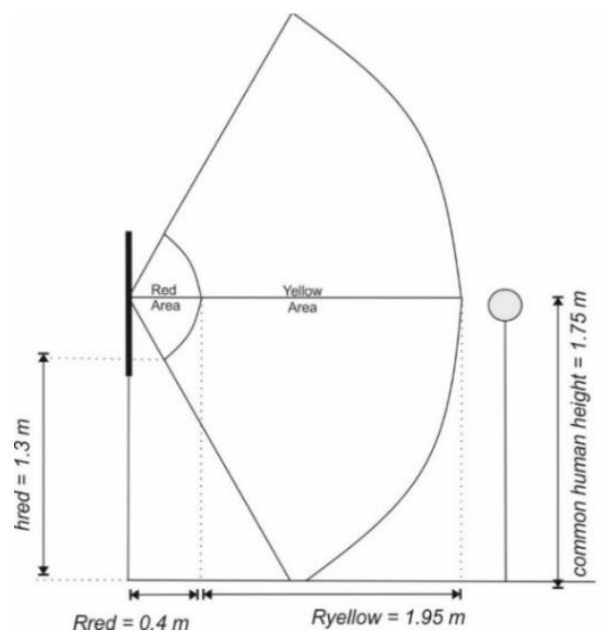

Figure 7. Estimated result for critical zone identification in the case of the UHF system. Since the antenna is omnidirectional the radiation is the same in 360 degrees

These zones are further extended to $0.67 \mathrm{~m}$ and 2.37, from the antenna for red and yellow zones, respectively when an uncertainty coefficient value of 1.6 is used. Table 6 summarises the predicted results for each case.

Table 6. Calculation of safety zone limits

\begin{tabular}{ccc}
\hline Case & R $_{\text {red }}$ & Ryellow \\
\hline UHF $(\mathrm{u}=1)$ & 0.4 & 1.95 \\
UHF $(\mathrm{u}=1.6)$ & 1 & 5 \\
VHF $(\mathrm{u}=1)$ & 0.47 & 1.32 \\
VHF $(\mathrm{u}=1.6)$ & 0.67 & 2.37 \\
$*$ All values in meters $(m)$ & \\
\hline
\end{tabular}




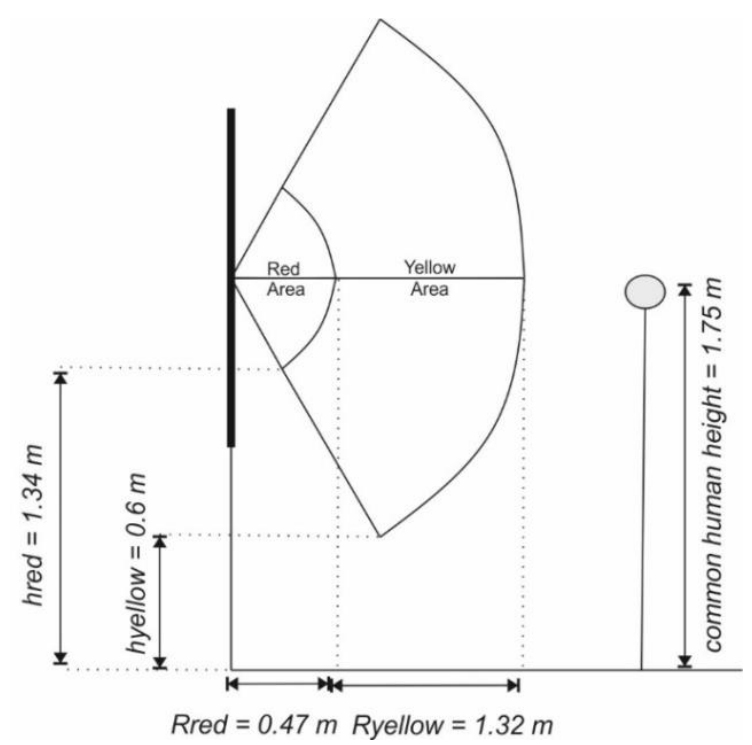

Figure 8. Estimated result for critical zone identification in the case of the VHF system. Since the antenna is omnidirectional the radiation is the same in 360 degrees

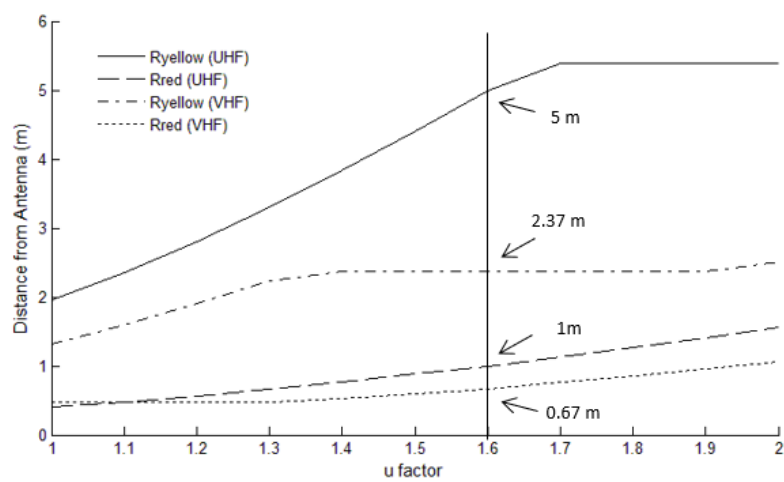

Figure 9. Calculation of safety zones for different $u$ coefficients

Going one step further we calculated the values of the safety zones for values of the coefficient $u$ in the range of 1 to 2 (according to FCC [5] a value of 2 corresponds to a highly reflective environment. This value was further adopted by IEEE for the evaluation of TV and FM radio broadcast systems). Figure 9 presents the results for both systems. It is shown that for values larger than the red area is slightly longer, but the yellow area presents a significant increase.

\section{DISCUSSION}

While numerical analysis indicated restricted areas up to 2 meters, the authors agree with Falsaperla et al. [20] that mitigation requirements are not very severe, due to limited use of RF systems and short duration of potential radiation exposure. Besides, there are no significant (other than thermal) impacts reported in the literature for short-term exposure for this frequency range. In that work, the authors address several technical - engineering, and organizational - administrative measures to ensure compliance with exposure standards. Following their approach, common engineering measures that must be adopted on board the seismic vessel are:

(1) Moving the antenna to a more protected environment, far from public access.
(2) Mount the antenna in a mast with a height larger than $h_{\text {red }}$ and $h_{\text {yelow. }}$.

(3) Installation of barriers and/or alarms close to the antenna.

Based on the fact that RF communications are a critical system onboard and most of the time engineering measures (such as leveling the antenna or transfer it to another point) are unable to take place, both due to space limitation and weather conditions, administrative control should be performed. In general, this kind of control measure is of low cost, and its effectiveness is strengthened by appropriate actions [18]. As a result, the following control steps should be followed by crew members and/or field engineers:

(1) Identification of every radiating source.

(2) Theoretical evaluation of its exposure.

(3) The region must be signed as an electromagnetic exposure area and the safety distance must be visible to all crew.

(4) Optimization of work-shifts to eliminate exposure time.

(5) Evolving personnel (operators, technicians, and medic) must be informed before working on restricted areas.

(6) Training/Information of all crew about the dangers and the appropriate protective actions that must be taken to eliminate their exposure.

Also, irregularities due to systems malfunction, such as corroded connectors or mismatched loads, etc., must be identified by dedicated periodic EM radiation measurement plans.

Although it is the responsibility of the operator to perform the above-mentioned steps, the active involvement of the MMO in the identification of the potential sources and even the theoretical evaluation and reporting of the calculated values could be crucial for the triggering of the proper control measurements. In any case, it is the MMO shift leader's responsibility to keep informed all observers in such a way that every observer to be exposed to the minimum possible radiation. Following the Health \& Safety Authority's instructions [21] the information shall include among others:

(1) potential risks and preventive measures taken

(2) how to detect and report signs of adverse health effects

(3) possibility of transient symptoms and sensations

(4) safe working practices to minimize risk from exposure to EMFs and

(5) use of personal protective equipment (PPE), if needed.

\section{CONCLUDING REMARKS}

A step towards the assessment of radiation exposure levels of two different radiofrequency communication systems installed onboard was presented. While results are valid for all crew on-board, we focused on Marine Mammals Observers, in the sense that only systems in the close vicinity of their working environment were analyzed. Single-source radiation was evaluated, as well as we did not consider any irregularities due to system malfunction. Despite these assumptions, the worst-case scenario of full power and continuous transmission of the system has been assumed. As a result, critical distances for the safety of both working personnel and common people have been reported. Special attention was given to the 
evaluation of the uncertainty introduced due to the specific operating environment of each system.

It was shown that for each evaluated system there are areas where Marine Mammal Observers should not enter due to the risk of over-exposure to Electromagnetic radiation. Engineering mitigation measures were briefly discussed, while administrative control measures were proposed through a detailed checklist.

A logical next step in our research would be the systematic evaluation of other, sources of RF radiation on-board, like RADARS, telemetry systems, compressors, etc. A similar modeling approach for RF systems operating in the HighFrequency range (HF) is currently underway since $\mathrm{HF}$ radiation on very high values may impact the central nervous system. Furthermore, quantification of risk associated with handheld RF communicating systems, based on measurements, is in progress. Preliminary results in both cases lead to significant findings.

\section{REFERENCES}

[1] Watkins, W.A. (1981). Activities and underwater sounds of fin whales. Sci. Rep. Whales Res. Inst, 33: 83-117.

[2] Stone, C.J. (2015). Implementation of and considerations for revisions to the JNCC guidelines for seismic surveys. Joint Nature Conservation Committee. JNCC Report No. 463b, JNCC, Peterborough, ISSN 0963-8091.

[3] House, I., Street, B. (2017). JNCC guidelines for minimising the risk of injury to marine mammals from geophysical surveys. Joint Nature Conservation Committee.

[4] DNV-GL Class Guideline. (2015). DNVGL-CG-0339: Environmental test specification for electrical, electronic and programmable equipment and systems. Germany.

[5] Mousa, A. (2011). Electromagnetic radiation measurements and safety issues of some cellular base stations in Nablus. Journal of Engineering Science \& Technology Review, 4(1): 35-42. https://doi.org/10.25103/jestr.041.05

[6] Kapurch, S.J. (2010). NASA Systems Engineering Handbook. Diane Publishing.

[7] Fields, R.E. (1997). Evaluating compliance with FCC guidelines for human exposure to radiofrequency electromagnetic fields. OET Bulletin, 65(10): 97-01.

[8] IEEE Standard. (2002). Standard 521: IEEE Standard Letter Designations for Radar-Frequency Bands. USA.

[9] International Commission on Non-Ionizing Radiation Protection. (1998). Guidelines for limiting exposure to time-varying electric, magnetic, and electromagnetic fields (up to $300 \mathrm{GHz}$ ). Health Phys., 74: 494-522.

[10] Sage, C., Carpenter, D.O. (2009). Public health implications of wireless technologies. Pathophysiology,
16(2-3):

233-246.

https://doi.org/10.1016/j.pathophys.2009.01.011

[11] IEEE Standard. (2005). Standard C95: Coordinating Committee 2. IEEE standard for safety levels concerning human exposure to radiofrequency electromagnetic fields, $3 \mathrm{kHz}$ to $300 \mathrm{GHz}$. USA.

[12] NATO Standard. (2003). STANAG, N. 2345-Evaluation and Control of Personnel Exposure to Radio Frequency Fields- $3 \mathrm{kHz}$ to $300 \mathrm{GHz}$.

[13] Electronic Communications Committee. (2003). Measuring Non-Ionising Electromagnetic Radiation $(9 \mathrm{kHz}-300 \mathrm{GHz})$. Recommendation adopted by the Working Group Frequency Management (FM).

[14] European Parliament Directive. (2013). 2013/35/EU: on the minimum health and safety requirements regarding the exposure of workers to the risks arising from physical agents (electromagnetic fields) (20th individual Directive within the meaning of Article 16 (1) of Directive 89/391/EEC) and repealing Directive 2004/40/EC. Official Journal of the European Union L, 179. Brussels, Belgium.

[15] IEC Standard. (2002). IEC Standard for 60945: Maritime navigation and radiocommunication equipment and systems-General requirements-Methods of testing and required test results.

[16] Lloyd's Register Type Approval Test Specification. (2015). Test Specification 1 - Performance and Environmental Test Specification for the following Environmentally Tested Products used in Marine Applications: Electrical Equipment, Control and Monitoring Equipment, Instrumentation and Internal Communication Equipment, Programmable Electronic Systems. The UK.

[17] DNV-GL Class Guideline. (2015). DNVGL-CG-0339: Environmental test specification for electrical, electronic \& programmable equipment and systems. Germany.

[18] IEC Standard. (2017). IEC 62232:2017: Determination of RF field strength, power density and SAR in the vicinity of radio communication base stations for the purpose of evaluating human exposure. Switzerland.

[19] Spandonidis, C.C., Ioannidi, K.C., Papadopoulos, N. (2013). Mathematical approach of electromagnetic interference analysis and safety radiation zones identification. Journal of Computations \& Modelling, 3(4): 25-40.

[20] Falsaperla, R., Spagnoli, G., Rossi, P. (2006). Electromagnetic fields: principles of exposure mitigation International journal of occupational safety and ergonomics, $12(2)$ : 195-200. https://doi.org/10.1080/10803548.2006.11076683

[21] Health and Safety Authority. Safety, Health, and Welfare at Work (Electromagnetic Fields): Regulations. 2016; S.I. No. 337 of 2016. Ireland. 